\title{
Dynamic Pattern Generation in Behavioral and Neural Systems
}

\author{
G. SCHÖNER AND J. A. S. KeLso
}

In the search for principles of pattern generation in complex biological systems, an operational approach is presented that embraces both theory and experiment. The central mathematical concepts of self-organization in nonequilibrium systems (including order parameter dynamics, stability, fluctuations, and time scales) are used to show how a large number of empirically observed features of temporal patterns can be mapped onto simple low-dimensional (stochastic, nonlinear) dynamical laws that are derivable from lower levels of description. The theoretical framework provides a language and a strategy, accompanied by new observables, that may afford an understanding of dynamic patterns at several scales of analysis (including behavioral patterns, neural networks, and individual neurons) and the linkage among them.

A LTHOUGH THE AMOUNT OF INFORMATION NECESSARY TO describe the individual states of neurons and muscles is very large, animals, nevertheless, possess a high degree of coordination. Indeed, coordinated motor activities-from walking to talking - present an example, par excellence, of the dynamic patterns found in nature. Yet the manner by which complex biological systems are coordinated to produce functionally specific ordered behavior or spatiotemporal patterns remains one of the great unsolved problems of biology (1). A major drawback is that the laws or principles governing pattern generation in biological systems are not known, though it would surely be significant to find them (2). In complex systems it is generally not possible, even if it were useful, to determine the detailed behavior of every degree of freedom. The problem is to select only the relevant parameters of the system, thereby reducing unnecessary information. An increasingly voiced concern among neuroscientists, for example, is that enormous advances in knowledge of cellular and synaptic phenomena have occurred in the last decade, but insights into the organizational principles of neural and behavioral function remain few $(3,4)$. The time may be ripe, therefore, to complement studies of cellular mechanisms with unifying concepts, particularly with regard to cooperative effects like coordination and pattern generation. Cooperative phenomena in nature are typically independent of the particular molecular machinery or material substrate that instantiates them $(5,6)$. For example, locomotion is fundamentally a rhythmically coordinated pattern shared by all animals that is realized by a wide diversity of anatomical structures and neural mechanisms. It is possible, therefore, that principles of coordination may lie at the

G. Schöner is a staff scientist at the Center for Complex Systems and J. A. S. Kelso holds the Glenwood and Martha Creech Chair in Science and is director of the Center for Complex Systems, Florida Atlantic University, Boca Raton, FL 33431. level of the patterns themselves, and that a focus on pattern (including the dynamical features of self-organization, stability, and adaptability) could provide the conceptual leap necessary to advance our understanding of biological coordination.

In this article, we aim to show that it is possible to understand behavioral pattern generation on several levels of description (kinematic, electromyographic, neuronal) by means of the concepts and tools of stochastic nonlinear dynamics. We will provide evidence, in certain cases, that once the essential macroscopic variables characterizing coordinated movement patterns are identified, it is possible to derive these patterns by cooperatively coupling individual microscopic components. In demonstrating that dynamical concepts are useful at several levels of observation, we wish to overcome the language barrier that currently exists among scientists who observe complex systems at very different scales of analysis. In the process, we seek to provide a minimal set of dynamical laws for pattern generation in complex, biological systems.

\section{The Dynamics of Pattern Formation: Concepts, Definitions, and Measures}

It is well known that spatial and temporal patterns in nonequilibrium physical and chemical systems can emerge spontaneously (68). This, "self-organized" (9) pattern formation is a collective phenomenon and results from the interaction of a large number of subsystems. To explain what we mean by self-organization in this context, a few theoretical concepts must be introduced.

Nonequilibrium systems generally obey dissipative dynamics. The term dissipative, as used here, means that many independent trajectories of the system with different initial conditions eventually approach each other in state space. That is, given sufficient time all trajectories will converge on a certain limit set, the attractor. Assuming that the relevant microscopic variables are known and can be lumped together in a (potentially very high dimensional) vector q, we may write down a quite general dynamic equation for such systems (10):

$$
\dot{\mathbf{q}}=\mathbf{N}(\mathbf{q}, \text { parameters, noise) }
$$

where $\mathbf{N}$ is, in general, a nonlinear function of the microscopic state vector $\mathbf{q}$. This function may also depend on a number of parameters representing, for instance, environmental conditions, as well as random forces that reflect the many degrees of freedom acting on the system but which are unaccounted for in the state vector $q$. The latter two dependencies will be discussed at length below because they are of decisive conceptual importance for the strategy we shall develop.

Typically, when parameters in Eq. 1 change continuously, the corresponding solutions of Eq. 1 also change continuously. However, there exist special critical points in parameter space, where the 


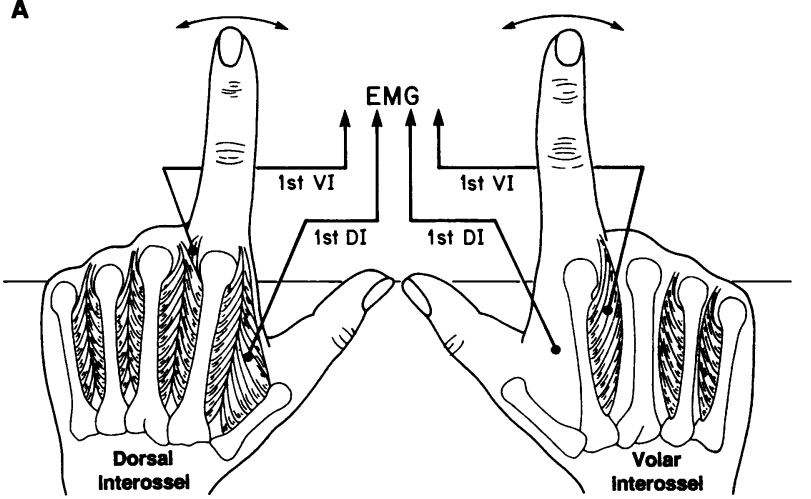

B

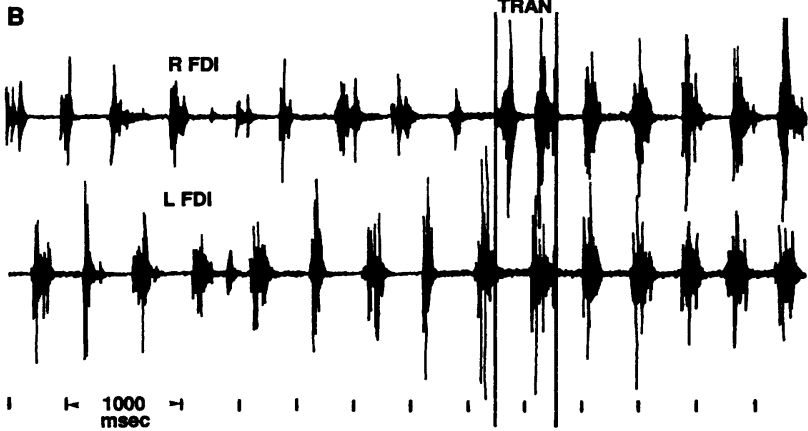

Flg. 1. (A) The experimental configuration for studying phase transitions. On a given trial the subject oscillates his or her index fingers bilaterally in the transverse plane, that is, abduction-adduction. Continuous finger displacement is sampled at $200 \mathrm{~Hz}$ by means of infrared light-emitting diodes attached to the finger tips. Electromyographic (EMG) activity of the right and left first dorsal interosseus (FDI) and first volar interosseus (FVI) is obtained by inserting platinum fine-wire electrodes. (B) EMG record from the FDI of the left and right index fingers. Initially the muscle bursts are anti-phase. At a critical frequency of oscillation, a spontaneous switch (TRAN) to an in-phase firing pattern occurs. (C) Representative time series showing position over time (upper trace) of the two index fingers as the control parameter, frequency, is increased in time and corresponding point estimate of relative phase (lower trace) based on the phase of one finger's oscillatory peak relative to the other. $\mathrm{ABD}$, abduction; ADD, adduction. solutions may change qualitatively or discontinuously. Such discontinuous changes are called nonequilibrium phase transitions and are frequently associated with the spontaneous formation or change of spatial or temporal patterns. For example, a system may have only homogeneous solutions for a range of parameters. Now, as environmental conditions are changed, spatially patterned solutions [for example, hexagons as in Bénard convection (11)] may suddenly appear. It is important to stress that relevant environmental changes can be completely unspecific to the pattern that emerges [in the above example, for instance, the control parameter (12) that crosses a critical point is just a change in temperature, which contains no information about the emerging spatial structure, its form, size, and so forth]. These patterns arise solely as a result of the dynamics of the system (that is, the function $\mathbf{N}$ in Eq. 1), with no specific ordering influence from the outside and no homunculus inside. Hence, the patterns are referred to as self-organized. Since dissipation is crucial to their existence and self-sustaining character, these patterns are also referred to as dissipative structures $(8)$. Well-known examples include the formation of convection patterns in fluid dynamics, the emergence of the coherent light field of the laser, and the formation of concentration patterns in certain chemical reactions (11).

How is the self-organization of patterns to be understood? In the theory of nonequilibrium phase transitions it is possible to show that close to critical points the system given by Eq. 1 may be completely described by much lower dimensional dynamics, specified in terms of only a few collective variables, the so-called order parameters (13), that characterize the emerging pattern. The consequent reduction in degrees of freedom, referred to as the slaving principle, has been given exact mathematical form for a large class of systems $(7,14)$. In this theory (known generally as synergetics), it becomes clear that the pattern formation is entirely due to the dynamic interaction of the many degrees of freedom in the system. In the absence of a thermodynamic interpretation of an equation of motion such as Eq. 1, it may be better to call these patterns dynamic patterns.

Is it possible to understand biological coordination and function

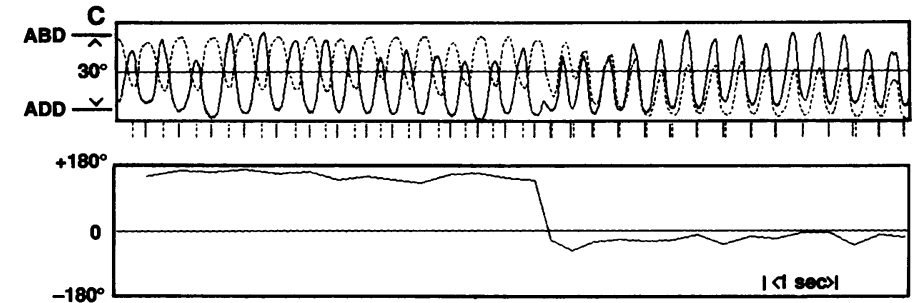

as dynamic patterns (15)? Obviously the path from microscopic to macroscopic is not as easily accessible in biological as it is in physical systems. To give meaning to an equation such as Eq. 1 is quite impossible. Therefore we begin with a disclaimer: understanding biological order will not be a simple "application" of the theories of nonequilibrium phase transitions (16). Nevertheless, we show here how it is possible to (i) establish quantitative and reproducible relations among observables in the form of laws, and (ii) make novel predictions that can be checked experimentally. We organize our results in the form of theoretical propositions that aim at a more general understanding of pattern formation, stability, and change in biological systems (16).

Proposition 1. Behavioral patterns (for example, neuronal, electromyographic, kinematic) can be characterized by collective variables (or order parameters), whose nature and dynamics are specific to biological functions and tasks.

Assume, for the moment, that we have found a set of collective variables characteristic of a biological pattern, that can be expressed by a low-dimensional vector $\mathbf{x}$. Underlying the dynamic pattern view is the assumption that $\mathbf{x}=\mathbf{x}(t)$, where $t$ is time, obeys a dynamical law (similar to Eq. 1, but now on a macroscopic level):

$$
\dot{\mathbf{x}}_{t}=\mathbf{f}\left(\mathbf{x}_{t}, \text { parameters, noise }\right)
$$

For a large class of functions $f$ special solutions of Eq. 2 -called attractors-exist (17). By definition an attractor is (asymptotically) stable, that is, all neighboring solutions converge in time to the attractor solution. The simplest attractor type is a stable fixed point, that is, a constant solution of Eq. 2, to which all neighboring trajectories converge (18). Another attractor type, important in the present context and perhaps for biology in general, is the limit cycle, a stable periodic solution of Eq. 2. Many more complicated attractor types exist, giving rise to very complex behavior of the solutions of Eq. 2 (19). Moreover, several attractors with different basins of attraction may coexist, a feature called multistability. The total set of solutions of Eq. 2 for given parameters is called a phase portrait, whereas the total number of possible phase portraits as parameters are varied is called a phase diagram (20). 
Fig. 2. A phase diagram of Eq. 3 in the parameter plane $(a, b)$. The transition line $(a=4 b)$ separates the monostable regime with an attractor at $\phi=0$, and the bistable regime with attractors at $\phi=0$ and $\phi= \pm \pi$. The insets illustrate the form of the potential in the mono- and bistable regimes. The control curve shows how the system approaches the transition line as the experimental parameter (frequency) is increased. [Reprinted from (27) with permission, copyright 1986, Springer-Verlag]

Proposition 2. Experimentally well-defined behavioral patterns correspond to stable collective states (or attractors) of the order parameter dynamics.

Several features of an observable pattern are described as follows: reproducibility, as revealed by independence from the detailed initial conditions; and stationarity (in a stochastic sense), as indexed through stability measures (see discussion of time scales below). At this point we can provide a quite general argument as to why the pattern dynamics of Eq. 2 should, in general, be nonlinear. The reason is that under given boundary conditions biological systems often exhibit multiple behaviors. In neural networks, for instance, this property is known as multifunctionality. In the language of dynamic patterns, such situations correspond to the existence of multiple stable states. Because of the dissipative character of Eq. 2, necessary to capture (asymptotic) stability, no linear dynamical system can account for the required multistability (21).

Let us now illustrate propositions 1 and 2 through an example from rhythmic movement patterns, which have been shown to represent a wide variety of coordinated behaviors in a very large number of biological systems (22) at different levels of observation (23). As a particular case consider the movement of two hands, operating at a common frequency. In such situations only a few modes of coordination-corresponding to phase-locked patternsare stably performed. One is an in-phase pattern, where the relative phase, $\phi$, is zero; the other is an anti-phase pattern (relative phase is $\pm \pi \mathrm{rad}$ ) $(24,25)$ (see Fig. 1). One can conceive of this case as a simplified experimental model for the locomotory gaits of trotting and galloping. A candidate collective variable that succinctly captures the dynamics of these coordinative patterns is the relative phase between the two rhythmically moving components. The observed in-phase and anti-phase patterns may be mapped onto point attractors at $\phi=0$ and $\phi=\pi$. Taking into account symmetries, the simplest dynamical system that accounts for the observed phase diagram may be given explicitly $(26,27)$ :

$$
\dot{\phi}=-\frac{d V(\phi)}{d \phi}+\text { noise }
$$

Thus a potential $V(\phi)=-a \cos (\phi)-b \cos (2 \phi)$ exists, which affords a visualization ( 7 ) of the solutions as the (overdamped) movement of a mass in an energy "landscape" defined by $V$. The attractors are thus the minima of $V$, whereas maxima of $V$ are unstable fixed points that separate different basins of attraction. In Fig. 2 a phase diagram of Eq. 3 is shown. The insets illustrate the form of the potential: on the upper left two minima at 0 and $\pm \pi$ exist (note that $\pi \equiv-\pi$ ); in the lower right only one fixed point $(\phi=0)$ is stable. In this simple experimental system, the relevant control parameter is the frequency of oscillation, which can be paced by the subject or paced externally by a metronome signal that the subject follows. Inertial or viscous loadings may also act as parameters (25). To account for the observed phase diagram, these parameters must be mapped onto the theoretical parameters $a, b$, and the noise strength in Eq. 3 (28).

It is possible to analyze this same behavior at another level of observation, the neuromuscular activities themselves. Fine-wire recordings from the agonist and antagonist muscles involved in the rhythmic movements of both limbs may be used to derive a measure of relative timing [the $\eta$-measure in (29)]. Such a measure directly reflects the relative phasing of the movements at the kinematic level. Point attractors (at $\eta=1$ and $\eta=0$ ) are shown to account for the observed patterns. A third level of observation (the kinematics of the individual limbs) will be discussed below.

Proposition 3. Loss of stability leads to change of behavioral pattern and is accompanied by a growth in relaxation time and enhanced fluctuations of the order parameter.

The real power of our approach lies in the central concept of stability. Stability can be measured in several ways: If a small perturbation drives the system away from its attractor, the time for the system to return to its attractor is independent of the size of the perturbation (as long as the latter is sufficiently small). This "local relaxation time," $\tau_{\text {rel }}$ (local with respect to the attractor), is therefore an observable system property that indexes the stability of the attractor state. The smaller $\tau_{\text {rel }}$ is, the more stable is the attractor. The case $\tau_{\text {rel }} \rightarrow \infty$ corresponds to a loss of stability. A second measure of stability is related to the noise sources indicated in Eq. 2. Any real system described by low dimensional dynamics will be composed of, and be coupled to, many subsystems. These act as stochastic forces on the collective variables, causing them to fluctuate. In the present context, the noise sources act as continuously applied perturbations and therefore produce deviations away from the attractor state. The amount of fluctuations as measured, for example, by the variance or standard deviation (SD) of $\mathbf{x}$ around the attractor state, is a measure of the stability of this state. The more stable the attractor, the smaller the mean deviation from the attractor state for a given strength of stochastic force. Without elaborating the details, a third measure of relaxation time may also be determined from fluctuation measures by determining the line width of the spectral density function (30).

All these stability measures have been used in studies of physical, chemical, and biochemical systems. Once an order parameter for behavioral and neuronal patterns is found, these observables also become accessible in biological experiments. For example, all three methods have been used to assess the stability of coordinated movement patterns. Perturbations have been introduced by applying a mechanical torque to one of the oscillating hands. Using interactive computer displays, we obtained an estimate of the relaxation time from the time of torque pulse offset until the relative phase time series stabilized at its pre-perturbation mean value. In this fashion, it was possible to discriminate patterns of different stability (31). Fluctuations in the patterns were measured by the SD of the relative phase time series (32), and again differences in stability were noted. An estimate of $\tau_{\text {rel }}$ from spectral line widths, technically more difficult, nevertheless showed results convergent with those obtained from directly perturbing the system (33).

One reason why stability is so important is that it can be lost. This is exactly what happens in nonequilibrium phase transitions. As a control parameter crosses a critical point the previously stable pattern becomes unstable, and the system switches to a different pattern that is stable beyond the critical point. The quite general predictions of nonequilibrium phase transition theory are a strong enhancement of fluctuations (critical fluctuations), and a strong increase in relaxation time (critical slowing down) as the transition is approached (7).

In the hand-movement experiments such a nonequilibrium phase transition was discovered as oscillation frequency was increased 
Fig. 3. (A) The average standard deviation (SD) of the relative phase as a function of the control parameter (frequency) for a set of ten experimental runs for a typical subject. Closed triangles refer to the in-phase pattern and open triangles to the anti-phase pattern. On a given run, the mean and SD are calculated for the last $3 \mathrm{sec}$ (600 samples) at a given frequency. [See (32) for details. Reprinted with permission, copyright 1986, North-Holland] (B) The mean relaxation time as a function of the control parameter (frequency) for a typical subject. Again closed triangles refer to the in-phase pattern and open triangles to the anti-phase pattern. The mean transition frequency for this subject was $2.21 \pm 0.17 \mathrm{~Hz}(N=30)$. [See (31) for details. Reprinted with permission, copyright 1987, North-Holland]

(Fig. 1). If the system was prepared initially in the anti-phase state, an involuntary, spontaneous switch to the in-phase mode occurred at a critical frequency (25). In the theoretical phase diagram (Fig. 2) this can be understood as a change in model parameters $a$ and $b$ along the line designated as the control curve, which crosses from the bistable regime (attractors at $\phi=0$ and $\phi= \pm \pi$ ) into the monostable regime (attractor at $\phi=0$ ). As the transition line is approached the anti-phase state loses its stability: that is, the minimum of the potential at $\phi= \pm \pi$ flattens out and finally disappears (turns into a maximum). The theoretical predictions of critical fluctuations and critical slowing down $(7,27)$ have been confirmed in a series of experiments $(25,29,31-33)$ (Fig. 3).

Another experimentally accessible feature-the so-called switching time-is contained in the stochastic order parameter dynamics. Switching time is the duration of the transient to the new state from the previous state that loses stability. The speed at which the system switches from the unstable to the new stable state depends on the level of noise in the system (34). As shown in Fig. 4, recent work has found excellent agreement between theoretical and experimentally determined measures of switching time, lending further support to the claim that the behavioral patterns-their stability and changefollow the stochastic order parameter dynamics Eq. 3 in some detail. There are other signatures of such qualitative changes of dynamics, such as hysteresis phenomena, addressed elsewhere (26, 29).

Propasition 4. Fluctuations in the dynamics of the order parameters reconcile the stability of behavioral patterns with the ability to change behavioral patterns by establishing time-scale relations that govern the switching dynamics among collective states.

Our claim is not that all behavioral changes are phase transitions. Phase transitions reveal, however, how the balance of fluctuations and stability determines a pattern's stability and flexibility. To explain this point in more detail, we have to overcome a conceptual difficulty related to the role of noise, the presence of which renders the interpretation of observed patterns as attractor states of a dynamical system nontrivial. This is particularly clear in multistable situations (in our example for the upper left part of the phase diagram of Fig. 2). If the system is initially prepared in one specific attractor it may, in finite time with finite probability, switch into the basin of attraction of another attractor as a result of the influence of stochastic perturbations (see Eq. 2). Indeed, the stationary probability distribution of the collective variable (which describes the system after a sufficiently long transient time) is, in general, a multimodal distribution that possesses some probability mass at the different coexisting attractors. How, then, is it possible to map observed patterns onto attractors of the order parameter dynamics (Eq. 2)? The key to this difficulty lies in the system's different time scales. Earlier we had introduced local relaxation times $\tau_{\text {rel }}$ as a measure of the time it takes the system to relax to an attractor, once it is nearby. A second time scale-the so-called time scale of observation $\tau_{\text {obs }}$ indicates the time frame over which statistical averages are per-

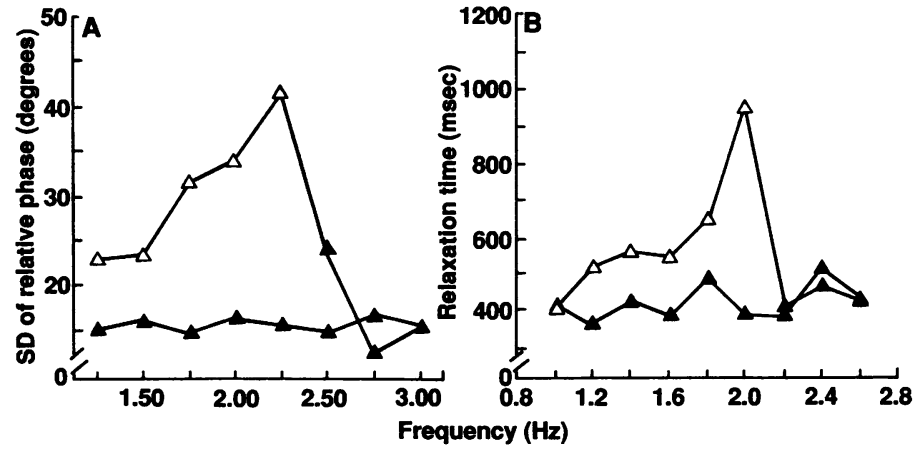

formed. A third time scale is the equilibration or global relaxation

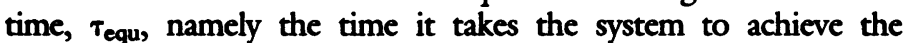
stationary probability distribution from a typical initial distribution. In a multistable situation, $\tau_{\text {equ }}$ is determined mostly by the typical time it takes to traverse from one basin of attraction into another (in the example of Fig. 2: to cross the potential hill between $\phi=0$ and $\phi= \pm \pi)$. If the foregoing time scales fulfill the following relation

$$
\tau_{\text {rel }}<<\tau_{\text {obs }}<<\tau_{\text {equ }}
$$

the interpretation of observed states as (local) attractor states is a consistent one. That is, the system has relaxed to a stationary state on the observed time scale, but is not yet distributed over all coexisting attractors according to the stationary probability distribution. When we refer to stationary states in an experiment, we mean that relation 4 is fulfilled. In other experimental situations, $\tau_{\text {obs }}$ may be much greater than $\tau_{\text {equ, }}$ as when a system is sampled over a long time and frequently changes state during the sampling period. In this case the underlying dynamics of multiple attractors will be evident from the form of the (stationary) probability distribution of the order parameter. In a bistable case with fixed-point attractors, for instance, the distribution will have two peaks at the two attractors and the width of these peaks will reflect the local stability of the attractors. For example, in studies of horse locomotion the frequency distribution of running speeds has three peaks that reveal the underlying gait structure (walk, trot, and gallop) (35). The limit $\tau_{\text {obs }}>>\tau_{\text {equ }}$ may be generally relevant to neurobiology (for example, when behaviors occur spontaneously).

As a state loses stability, its local relaxation time increases until eventually at the phase transition, the relation 4 is violated and switching takes place. The nature of the transition (for example, whether critical fluctuations will be observed) depends on a fourth time scale, the time scale for parameter change, $\tau_{p}$. For $\tau_{p} \gg \tau_{\text {equ, }}$, the typical situation in physical systems, critical phenomena (for example, critical fluctuations or critical slowing down) can be observed only in special cases, that is, when a symmetry is broken (5). In contrast, in biological systems, $\tau_{p}$ may often be smaller than $\tau_{\text {equ. }}$ In such cases critical phenomena are expected irrespective of symmetry $(33,34)$. Importantly, however, all the different time scales are measurable. In some cases, $\tau_{o b s}$ and $\tau_{p}$, this is quite obvious. In others, such as $\tau_{\text {equ, several measurement techniques }}$ exist (as in the case of $\tau_{\text {rel }}$ discussed above). One direct measure is the so-called mean first-passage time (MFPT) among different attractors, that is, the average length of time before the system first changes state (30). The mean switching time also contains information about $\tau_{\text {equ }}(27)$. In our experiments (31) the MFPT was determined indirectly from parameter fits of fluctuation and relaxation time data, enabling a direct test of the relation 4 and its breakdown at the phase transition. Switching indeed occurred as the time scales relation 4 was violated.

Proposition 5. In a number of specific cases, collective states (for 
example, multiple phase and frequency-lockings) arise from a coupling of self-excited nonlinear (limit cycle) oscillatory processes.

We return, finally, to the question of levels of description in the context of our experimental example. It is possible to study the system on yet another level of description, namely that of the individual limb's dynamical behavior. Thus we may choose each limb's position $x_{i}$ and velocity $\dot{x}_{i},(i=1,2)$ as collective variables (collective now with respect to the next lower level of description, for example, the coordinated activity of agonist and antagonist muscles). The observed behavioral pattern [rhythmic movement with reproducible amplitude-frequency, frequency-velocity relationships (36)] is mapped onto an attractor of $\left(x_{i}, \dot{x}_{i}\right)$, in this case a limit cycle. Again, with a small set of specific assumptions, an explicit nonlinear oscillator model captures a number of experimental features (36). The stability of this attractor was measured by means of perturbation techniques (37) similar to those described for the coordinated case. Through a detailed phase resetting analysis of these perturbation experiments, the system's dynamics were demonstrated to be autonomous, that is, not explicitly time-dependent (37). Once again then, a potentially complex behavior, observable in many ways, can be mapped onto a simpler dynamical description whose consistency can be checked experimentally. An understanding of the component dynamics, in the sense of dynamic patterns, can thus be reached.

The two levels of description that are understood dynamically (component oscillators and relative phase dynamics) can be related by introducing coupling functions among the individual components. In this case, it was possible to derive mathematically (with certain approximations) the relative phase dynamics Eq. 3 from the coupled component oscillators (26). A significant feature of the Haken, Kelso, and Bunz model (26) is that the experimentally observed phase transition arises-as a result of the nonlinear coupling structure-through a simple change of the oscillation frequency. It is important to note that the coupling functions are quite unspecific to the patterns of coordination that emerge. Several functional forms give rise to the same pattern of phase-locking (26). Also, changes in coordinative pattern can be brought about by keeping the coupling function constant and changing only the eigenfrequencies of the component oscillators (26). Thus the present theory provides a conceptual framework for understanding how very similar patterns may arise from a variety of mechanisms.

\section{Pattern Generation in Neurobiological Systems}

Let us step through the dynamic pattern approach-from collective variables and parameters, to pattern stability and change, and finally to relations among levels of description-pointing out the relations between these concepts and existing neurobiological work, suggesting in some cases a different viewpoint, and posing some new questions that arise from "embedding" neuronal data into this strategy. We limit discussion to neuronal behavior studied electrophysiologically both at the network level and the biophysical level of the individual neuron, during rhythmic and discrete animal actions. Of course, given the size of the literature, it is impossible to be inclusive.

Collective variables. Many patterns of activity in well-defined (mostly invertebrate) neural networks have been characterized sufficiently well to be given a name, often associating them with a behavior (38). Terms such as "swimming" central pattern generator (CPG), "flight" CPG, and "locomotor" CPG - though not agreed upon by everyone-reflect the fact that a given pattern is reproducible, is stationary over a certain amount of time, and can be characterized well enough to differentiate it from other neural

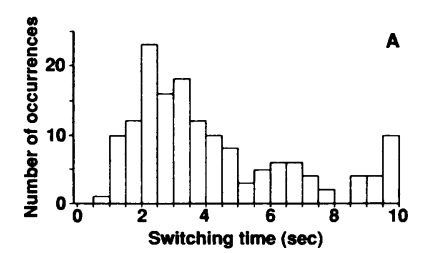

Fig. 4. (A) The empirically obtained distribution of switching times (time from last change of control parameter, frequency, to the completion of switching) from all subjects and all trials in (31). [Reprinted with permission, copyright 1987 , North-Holland] (B) The probability density of switching time as calculated theoretically from the stochastic dynamic theory (Eq. 3) by numerically integrating the corresponding Fokker-Planck equation. We note that no parameters were adjusted for this measure as all parameters could be determined from the mean, variability, and relaxation time measures. [See (27) for details. Reprinted with permission, copyright 1986, Springer-Verlag]

patterns generated by the same network. The relation of a neural pattern to an associated behavior is often established through a series of experiments with increasingly more isolated preparations. The behavior itself can be characterized quantitatively (39). A dynamic pattern analysis of the behavioral pattern that includes information about biological function can serve as an excellent guide in the quest for collective variables on the neural pattern level. An example in this direction is work (39) on Pleurobranchaea, in which temporal relationships among neurons are studied in relation to different feeding behaviors whose temporal characteristics have functional significance.

Obviously, rhythmic behaviors and associated neural patterns come closest to the dynamical theory we have developed above. Indeed, our main experimental example in human motor behavior will look familiar to many neurobiologists, in particular if described using electromyographic measures (29). In fact, typical phenomena of temporal ordering are abundant in the neuronal pattern generator literature, for example, synchronization and desynchronization, frequency-locking, phase-locking, fixed relation of lengths of firing bursts, and so forth (40). An important point is that these oscillatory and ordered phenomena are expressly collective. For example, a network of identified neurons in the buccal ganglion of Helisoma (the so-called cyberchrons) shows cyclically patterned activity when any one of the cells is stimulated, but fails to do so when the coupling among the cells is reduced experimentally (41). For such rhythmic activity, "discriminators" on the neural level typically refer to the temporal order found in the neural pattern, quantitatively evaluated through measures of relative phase and latency among components, neuronal burst frequency and frequency differences among neurons, and so forth (42). Such findings hint strongly that collective variables for temporal order in behavior are quite adequate also on the neural level (33). In fact, it is astonishing that of all the possible neuronal patterns available, only a few kinds of temporal order appear to be used. This amounts to tremendous information compression, sometimes referred to as a "degeneracy in the code for any pattern" (43). Thus, relative timing measures among neuronal bursts characterize different behavioral patterns: (i) within a species; (ii) across species; and (iii) within and across levels of development - even when changes in form [such as larval instars (44)] are dramatic. Although somewhat outside the present context, it is worth mentioning that recent observations on macroscopic neural activity [such as EEG in humans (45) and multi-electrode studies in monkeys (46)] reveal the existence of low-dimensional collective variables. With new computational tools from nonlinear dynamics such as the correlation dimension (47), surprisingly small numbers of degrees of freedom were sometimes found $(45,46)$. Such results 
suggest more qualitatively that the collective dynamics of underlying neural processes are low-dimensional.

Control parameters. Much is known about the parameters and surrounding conditions that influence neural patterns (for example, the "triggering" capability of tonic excitation, the "switching" induced by sensory inputs, and so forth). In a dynamic pattern context the main question concerns the relevant control parameters that promote qualitative changes in neuronal patterns. The problem of identifying such control parameters is nontrivial. Often pattern change occurs spontaneously or is brought about by changing an elicitation procedure rather than by purely parametric manipulation. It is highly desirable, therefore, to describe the elicitation process itself in dynamic terms (for example, typical time for elicitation process, variables that measure the "intensity" of the pattern during elicitation). In some cases, parameters that influence the elicitability of a pattern seem to be known. For example, the presence or absence of leg contact changes the function of "trigger" interneurons associated with cockroach flight initiation (49). The concentration of serotonin changes the elicitation threshold for rhythmic feeding patterns in Helisoma (50). Weak, short durational sensory inputs evoke reflex withdrawal in Tritonia (51). Intense, long durational input evokes swimming in the same creature-apparently because it depolarizes a cerebral cell, C2 (51).

Stability. Where collective variables for neuronal patterns are known, relaxation times and fluctuation measures are well-defined and open to observation. The key step is to link such measures to the concept of stability. Qualitatively speaking, the reproducibility of neural patterns attests to their stability. The existence of an underlying relaxation process becomes obvious, however, if the relaxation time becomes very long. Mpitsos and Cohan (52) reported recently what they call "history" effects in the feeding patterns of Pleurobranchaca. They showed that a motoneuron that initially fires in phase with a buccal ganglion nerve root (R3) in the primary (feeding) pattern switches to an anti-phase relation with the same neuron during a bout of vomiting. After the overall features (in terms of neural firing patterns) returned to the primary pattern, this same motoneuron retained its anti-phase relation for several minutes, then took on another burst pattern with doubled firing rate (an intermediate metastable state) before relaxing to its originally prepared inphase relation with $\mathrm{R} 3$. A finite relaxation time after parameter change, or a finite time needed for eliciting a pattern reflects a relaxation process [see also figure 5 of Getting and Dekin (51) for a recent example].

Fluctuation measures have been used in the past, though seemingly for more technical reasons. Although not interpreted as such, Wyman's (53) phase histograms of neural impulses during blowfly flight clearly show differing degrees of stability. But collective variables corresponding to the flight pattern were never identified. Relaxation time and fluctuation measures of stability could also serve as an important pattern discriminator, especially in cases where the mean state does not change. In studies of the development of the locust flight pattern (44), for example, the mean state as measured by relative phase does not seem to change much during development. However, stability-as observed through the variance of distributions of relative phase-does appear to distinguish developmental stages, although this feature, unfortunately, was not tested statistically.

Pattern change. The phenomenon of multifunctionality-a multiplicity of patterns generated by the same set of neuronal elementshas been a major conceptual challenge for a number of years (54). A recent example comes from observations by Mpitsos and Cohan (52) of different phase relationships among the same neurons in the buccal ganglion of Pleurobranchaea during egestion and ingestion behavior. It is evident that function is not rigidly coded into the structure of the neural network involved. A similar view has been expressed recently in contexts such as neuronal development (55) and central nervous system plasticity following peripheral injury (56).

Theoretical explanations of multifunctionality within the more orthodox, circuit-analysis-oriented strategy include the multipartite central pattern generator concept (57) and the polymorphic network concept (51). From a dynamic pattern perspective, however, such multifunctionality appears as a natural phenomenon, to be understood in terms of multistability. The coexistence of different stable states in a complex system is to be recognized as a commonly occurring, if not universal event. Spontaneously occurring episodes of changing neural patterns are indicative of a time scales relation, in which switching among attractors is observable. In such cases, once the collective variables and the attractor state corresponding to different patterns are identified, a detailed test is possible. Assessing the stabilities of the different patterns, measuring switching times, as well as global time scales (MFPTs)-as we demonstrated earliercan help establish theoretical understanding.

A crucial test of the dynamic pattern theory involves a study of phase transitions among neural patterns. Thus, if a parameter can be found that controls the switching among patterns, then loss of stability can be directly measured. The aim of such an undertaking is neither to find the anatomical locus of a "switch" nor to find a new mechanism for switching, but rather to show that pattern stability and pattern change are a consequence of the balance between stable dynamic states and fluctuating influences.

Relation of levels. In contrast to network behavior, the single neuron level appears to be understood in terms closer to the dynamic pattern spirit (58). On the experimental side, endogenously oscillating neurons have been studied with phase-plane techniques that have revealed the essential limit-cycle character of these neurons, if only over a few cycles of their oscillation (59). Perturbation techniques have also been employed in the phase resetting paradigm $(59,60)$. Such techniques could, however, be used to measure the stability of neuronal limit cycles. In addition, bifurcations from the rest state to limit-cycle behavior and onward to chaos have been experimentally observed in electrically stimulated giant squid axons (61). A model of a forced, nonlinear oscillator reproduces the squid axon data beautifully. If the dynamic pattern analysis outlined here were achieved on the network level, the linkage of levels of observation is possible in a way that parallels our studies of human interlimb coordination. Such theoretical attempts do exist (62), but a chief problem arises in defining the relevant network property to be derived. Similarly, the huge literature on biological oscillators (63) is more modeling oriented in the sense that a set of oscillator equations is explored in order to simulate certain biological properties. In our opinion, a major benefit of the dynamic pattern approach is its primary emphasis on the identification of order parameters and their dynamics.

One further challenge is worth noting. Recent findings in experimental neurobiology reveal that well-understood neural circuits show a surprising degree of plasticity (64). From this work, we see an important link between structure and function emerging. For example, a neurotransmitter (serotonin) may regulate growth during regeneration (65). Similarly, electrical activity can suppress neurite growth (66). Although a detailed mathematical understanding of the structure-function relation is a very amibitious goal, it nevertheless appears to be a possibility within the dynamic pattern view. For example, in the field of morphogenesis, spatial pattern formation owing to dynamic instabilities has been found in systems [such as the slime mold (67)] for which reasonable models of the underlying biochemistry exist (68). More generally, the same pattern may be obtained from subsystem dynamics that have different 
couplings, and multiple patterns may be produced by the same set of components and couplings. An invariance of function under change of material substrate (if by that we mean a reconfiguration of the connections or couplings among neural elements) is an intrinsic feature of the dynamic pattern approach.

\section{Concluding Remarks}

Using a strictly operational approach in which theory and experiment go hand in hand, we have shown that temporally coordinated behavioral patterns (their stability and change) may arise in a purely self-organized fashion. For such patterns, the predictions of nonequilibrium phase transition theory [synergetics (7)] have been confirmed to a remarkable degree. Because temporal order is a common, if not universal characteristic of living things (5), it may be the ideal paradigm to expose synergetic features at both macro and micro levels of description. Many of the features of observed behavioral patterns, including phase- and frequency-locking, multistability, loss of stability, and so forth, are common also to neuronal patterns generated by many species. By linking functionally specific dynamics at different scales of observation, our approach offers a way to relate macroscopic behavioral levels to more microscopic physiological levels. Thus the central concepts and tools of dynamic patterns may provide a conceptual framework for understanding central pattern generators (57) and their frequently cited behavioral counterpart, the motor program (69). As a formal metaphor, the motor program might usefully consider dynamical aspects (stability, loss of stability, time scales relations, and so forth).

Our approach also shares some common features with recent work on artificial neural networks that stresses the computational capabilities of collectives of "neurons" (70). Unlike the present operational attitude, however, the linkage between theory and experimental observables in neural network modeling is neither a prerequisite nor a necessary source of constraint. Most neural network modeling starts with model neurons and circuits and then attempts to produce various learning, memory, and pattern recognition schemes. For instance, prescribed sets of states can be made stable states of such networks that correspond to patterns or content-addressable memories (70). It is not clear, however, how to define such prescriptions a priori. Dynamic pattern theory, on the other hand, places a high priority on the identification of order parameters for patterns. This proves to be crucial for extensions of the approach that consider the influence of perception, memory, and learning on behavioral patterns (16) and how these, too, may be synthesized.

\section{REFERENCES AND NOTES}

1. H. H. Pattee, in Topica in the Pbilosopby of Biology, M. Grene and E. Mendelsohn, Eds., Boston Studies in Pbilosoplyy of Science (Reidel, Boston, 1976), vol. 27, Pp. 153-173.

2. See the commentaries in (4).

3. T. H. Bullock, in Simple Networks and Behavior, J. D. Fentress, Ed. (Sinauer, Sunderland, MA, 1976), pp. 52-60; K. G. Pearson, in Comparative Neurobiology, M. J. Cohen and F. Strumwasser, Eds. (Wiley, New York, 1985), pp. 225-244. 4. A. I. Selverston, Bebav. Brain Sai. 3, 535 (1980).

5. P. W. Anderson, Science 177, 393 (1972).

6. H. Haken, Rev. Mod. Pbys. 47, 67 (1975).

7. $\longrightarrow$, Symergetios-An Introduction (Springer-Verlag, New York, ed. 3, 1983).

8. G. Nicolis and I. Prigogine, Self-Organization in Nonequilibrium Systems (Wiley, New York, 1977).

9. The word self-organization has earlier uses, of course, in theoretical studies stemming from cybernetics and abstract automata [see, for example, M. C. Yovits and S. Cameron, Eds., Self Organizing Systems (Pergamon, New York, 1960); H. von Forster and G. W. Zopf, Eds., Principles of Self-Organization (Pergamon, New York, 1961)]

10. H. Haken, Ann. N.Y. Acad. Sci. 316, 357 (1979).

11. For a discussion of these and many other examples see ( 7 ) and references therein.
12. Parameters that lead the system through critical points are called control parameters. No connection with control theory is implied.

13. A misnomer, perhaps, since they are variables, not parameters. The term stems from the phenomenological theory of equilibrium phase transitions by L. D. Landau, Phys. Z. Sowjet. 11, 545 (1937).

14. H. Haken, Advanced Symergetios (Springer-Verlag, New York, 1983).

15. Neurobiologists may be aware of earlier attempts in this direction [see, for example, A. K. Katchalsky, W. Rowland, R. Blumenthal, Eds., Dymamic Patterns of Brain Cell Ascemblies (MIT Press, Cambridge, 1974), with many references]. In contrast to some of these more modeling-oriented works we would like here to stress the operational aspect.

16. J. A. S. Kelso and G. Schöner, Springer Proc. Pbys. 19, 224 (1987); G. Schöner and J. A. S. Kelso, Biol. Cybern. 58, 71 (1988); G. Schöner and J. A. S. Kelso, in Dynamic Pattems in Complax Systems, J. A. S. Kelso, A. J. Mandell, M. F. Shlesinger, Eds. (World Scientific, Singapore, in press).

17. This is the same notion as mentioned at the beginning of this section, but now relating to a much lower dimensional space. For tutorials on some mathematical concepts used here see, for example, chapters 5 and 6 of (7) or R. H. Abraham and C. D. Shaw, Dymamios-The Geometry of Bebavior (Aerial Press, Santa Cruz, 1982). We give only a very brief summary of the notions most pertinent to our discussion.

18. Point attractors have been used to represent computational operations performed by artificial neural networks [for example, content-addressable memory, see J. J. Hopfield, Proc. Natl. Acad. Sci. U.S.A. 79, 2554 (1982) for a recent example]

19. It is well known that such low-dimensional dynamical systems are capable of producing extremely complex and interesting behavior, including deterministic chaos. For an introduction, see, for example, H. G. Schuster, Deterministic ChaosAn Introduction (Physik Verlag, Weinheim, West Germany, 1984).

20. Note the two different meanings of the word phase. The phase portrait refers to the flow of a dynamical system as observed, for example, through phase plane trajectories. The word phase in "phase diagram" derives from thermodynamic phase. More generally, a phase diagram defines regions in parameter space that do not exhibit qualitative change of the dynamics as well as the boundaries across which such changes occur. Although these terms are sometimes used ambiguously in the literature, we shall retain the above definitions.

21. V. I. Amold, Ordinary Differential Equations (MIT Press, Cambridge, MA, 1973).

22. Studied, as such, in many species (invertebrate and vertebrate), involving different limbs and different biological functions by Erich von Holst in the 1930s. See E. von Holst, Collected Worts (Univ. of Miami Press, Coral Gables, 1973).

23. G. Wendler, J. Comp. Physiol. 88, 173 (1974).

24. J. A. S. Kelso, K. G. Holt, P. Rubin, P. N. Kugler, J. Motor Behap. 13, 226 (1981).

25. J. A. S. Kelso, Bull. Psychon. Soc. 18, 63 (1981); Am. J. Physiol. 246 (Regulatory Integrative Comp. Physiol. 15), R1000 (1984). Earlier, L. Cohen [Percept. Mot. Skills 32, 639 (1971)] observed transient behavior between the two coordinative modes, but did not manipulate frequency and hence did not establish the transition.

26. H. Haken, J. A. S. Kelso, H. Bunz, Biol. Cybern. 51, 347 (1985).

27. G. Schöner, H. Haken, J. A. S. Kelso, ibid. 53, 247 (1986).

28. Under standard assumptions the noise term in Eq. 3 is Gaussian white noise, whose variance, $Q$ is the noise strength parameter. For details see (27).

29. J. A. S. Kelso and J. P. Scholz, in Complax Systoms-Operational Approaches, H. Haken, Ed. (Springer-Verlag, New York, 1985), pp. 124-149.

30. See, for example, C. W. Gardiner, Handbook of Stochastic Methods (Springer-Verlag, New York, 1983).

31. J. P. Scholz, J. A. S. Kelso, G. Schöner, Plyys. Lett. 123A, 390 (1987).

32. J. A. S. Kelso, J. P. Scholz, G. Schöner, ibid. 118A, 279 (1986).

33. J. A. S. Kelso, G. Schöner, J. P. Scholz, H. Haken, Phys. Scr. 35, 79 (1987).

34. R. Landauer, J. Appl. Phys. 33, 2209 (1962); for a more recent review see R. Landauer, Ann. N.Y. Acad. Sci. 316, 433 (1979).

35. D. F. Hoyt and C. R. Taylor, Nature (London) 292, 239 (1981).

36. B. A. Kay, J. A. S. Kelso, E. L. Saltzman, G. Schöner, J. Exp. Psychol.: Hum. Perc. Perf. 13, 178 (1987).

37. B. A. Kay, thesis, University of Connecticut, Storrs, 1986.

38. See F. Delcomyn, Science 210, 492 (1980); S. Grillner, ibid. 228, 143 (1985); and (4).

39. Examples of quantitative analysis of behavior are L. I. Mortin, J. Keifer, P. S. G. Stein, J. Nexroplysiol. 53, 1501 (1985) in the turtle scratch reflex and R. P. Croll and W. J. Davis, J. Comp. Pbysiol. 145, 277 (1981) for feeding behavior of Plewrobranchaca.

40. Some recent examples are the contributions of part I of A. I. Selverston, Ed., Model Neural Networks and Bebavior (Plenum, New York, 1985); see also (23).

41. S. B. Kater, Amer. Zool. 14, 1017 (1974); C. R. S. Kaneko, M. Merickel, S. D. Kater, Brain Ras. 146, 1, (1978).

42. R. P. Croll, W. J. Davis, M. P. Kovac, J. Newrosci. 5, 48 (1985) is an excellent example, but there are many more than we can list.

43. W. B. Kristan, Jr., in Information Processing in the Nervous System, H. M. Pinsker and W. D. Willis, Jr., Eds. (Raven, New York, 1980), pp. 285-312.

44. P. A. Stevenson and W. Kutsch, Naturwisenschaften 73, 741 (1986).

45. See contributions of A. M. Albano et al., pp. 231-240; A. Babloyantz, pp. 241245; and S. P. Layne, G. Mayer-Kress, J. Holzfuss, pp. 246-256, all in G. MayerKress, Ed., Dimension and Entropies in Chaotic Systems (Springer-Verlag, New York, 1986).

46. P. F. Rapp, I. D. Zimmerman, A. M. Albano, G. C. de Guzman, N. M. Greenbaun, Plyss. Lett. 110A, 335 (1985).

47. P. Grassberger and I. Procaccia, Pbysica D9, 189 (1983).

48. See G. Mayer-Kress, S. D. Layne, Anr. N.Y. Acad. Sci. 504, 62 (1987).

49. R. E. Ritzmann, M. L. Tobias, C. R. Fourtner, Science 210, 443 (1980).

50. S. B. Kater, private communication.

51. P. A. Getting and M. S. Dekin, in (40), p. 3. 
52. G. J. Mpitsos and C. S. Cohan, J. Neurobiol. 17, 517 (1986).

53. R. Wyman, Biophys. J. 5, 447 (1965); J. Newrophysiol. 29, 807 (1966).

54. See, for example, D. M. Wilson, J. Exp. Biol. 39, 669 (1962); J. L. Ayers and W. J. Davis, I. Comp. Physiol. 115, 1 (1977).

55. S. S. Easter, Jr., D. Purves, P. Rakic, N. C. Spizer, Science 230, 507 (1985)

56. M. M. Merzenich and J. H. Kaas, Trends Newrosci. 5, 434 (1982).

57. S. Grillner, in (38)

58. For review see, for example, A. C. Scott, Neurophysios (Wiley-Interscience, New York, 1977); R. J. MacGregor and E. R. Lewis, Neural Modeling (Plenum, New York, 1977).

59. H. M. Pinsker and J. Bell, Biol. Cyberm. 39, 211 (1981).

60. A. T. Winfree, Science 197, 761 (1977)

61. K. Aihara, T. Numajiri, G. Matsumoto, M. Kotani, Phys. Lett. 116A, 313 (1986).

62. H. R. Wilson and J. D. Cowan, Kybernetik 13, 55 (1973); Y. Yamaguchi, K. Kometani, H. Shimizu, J. Theor. Biol. 82, 231 (1980); A. H. Cohen, P. J. Holmes, R. H. Rand, J. Math. Biol. 13, 345 (1982); see also (58) for review.

63. For a recent annotated bibliography see N. Kopell, J. Stat. Phys. 44, 1035 (1986). 64. See S. B. Kater, in (40), Pp. 191-209; and E. Frank, Tronds Newrosci. 10, 188 (1987) for review.

65. P. G. Haydon, D. P. McCobb, S. B. Kater, Science 226, 561 (1984).
66. C. S. Cohan and S. B. Kater, ibid. 232, 1638 (1986).

67. G. Gerish and B. Hess, Proc. Natl. Acad. Sai. U.S.A. 71,2118 (1974); A. Goldbeter and O. Decroly, Am. J. Physid. 245, R478 (1983)

68. A. Gierer and H. Meinhardt, Kybermetik 12, 30 (1972).

69. See V. B. Brooks, in Posture and Movement, R. E. Talbott and D. R. Humphrey, Eds. (Raven, New York, 1979), pp. 13-49; R. A. Schmidt, Motor Control and Learning (Human Kinetics, Champaign, 1982); S. W. Keele, in Handbook of Physiology, Motor Control, V. B. Brooks, Ed. (Williams \& Wilkins, Baltimore, 1981), vol. 2, pp. 1391-1414.

70. J. J. Hopfield, in (18); technical comments by G. A. Carpenter, M. A. Cohen, S. Grossberg; T. Kohonen and E. Oja, G. Palm, in Science 235, 1226 (1987)

71. This research is supported by a NIMH (Fundamental Neurosciences Branch) grant MH42900-01 and a joint contract NO0014-87-G-0156 from the U.S. Office of Naval Research (Physics Division and Integrated Biology Program) and the Air Force Office of Scientific Research (Artificial Neural Networks Program). G.S. was supported by a Forschungsstipendium of the Deutsche Forschungsgemeinschaft. We thank S. B. Kater and C. S. Cohan for intensive discussion and encouragement and G. Zimmermann for first bringing us together with these neurobiologists. We appreciate comments on an earlier version from $\mathrm{H}$. Cruse, J. Dean and B. Tuller.

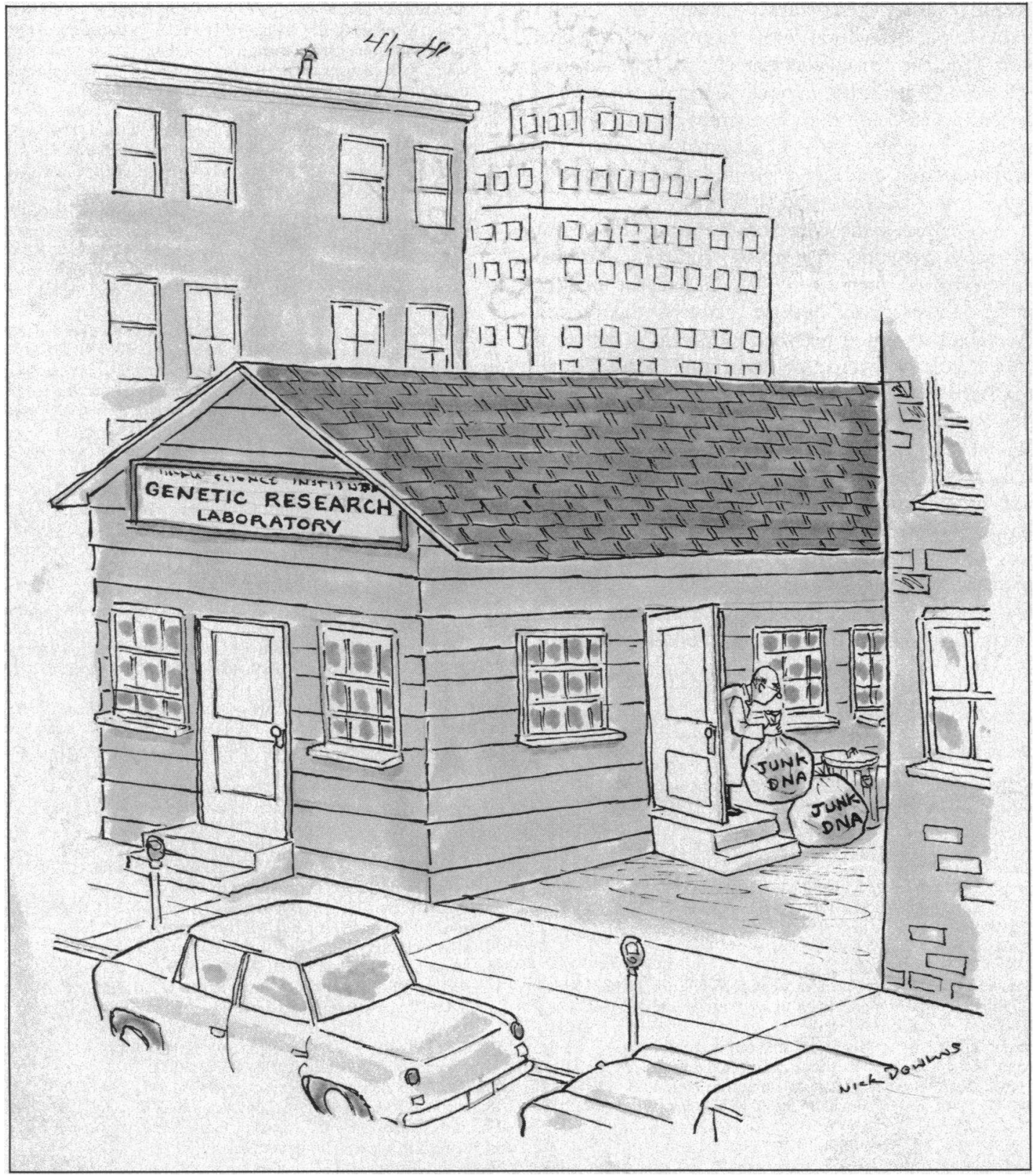

